\title{
The sensorless control strategy of UAV rotor motor in full speed range
}

\author{
Siyuan $\mathrm{Ma}^{1}$, Xiang Yu${ }^{1}$, Yunjun Guo ${ }^{1 *}$, Rong $\operatorname{Ran}^{1}$ \\ ${ }^{1}$ National Key Laboratory of Science and Technology on Vessel Integrated Power System, Naval University of Engineering, 430033 , \\ Wuhan, China
}

\begin{abstract}
UAV (Unmanned Aerial Vehicle) has broad application prospects in various fields. In order to meet the needs of stability and efficiency during flight, a surface-mounted permanent magnet synchronous motor is used as its rotor motor, and a position sensorless control technology is used to control the rotor motor with high efficiency and high dynamic response. This paper proposes a position sensorless control strategy in the full speed range for the actual application requirements of UAV. In low speed ange, I/F control strategy with speed-open-loop and current-closed-loop is. In medium and high speed range, a sliding mode observer is adopted to provide estimated rotor position and speed. To achieve smooth switching from I/F control stage to speed closed-loop stage, a switching algorithm is proposed. The feasibility of the proposed hybrid control strategy is verified by simulation results.
\end{abstract}

\section{Introduction}

With the development of technology, UAV systems have gradually integrated into all aspects of social development and become indispensable civilian equipment and military equipment[1]. The rotor motor of the aircraft adopts a surface permanent magnet synchronous motor, which has the advantages of high power density, high efficiency, wide speed range, light weight, and small size. Permanent magnets are used to replace the field winding excitation in ordinary electric-excited synchronous motors. It has a simpler structure and stronger reliability. With the performance improvement and cost reduction of permanent magnet materials, permanent magnet synchronous motors have been widely used in recent years in many fields such as rail transit, aerospace science and technology, and shipbuilding industry.

During the flight, the aircraft will face the complicated navigation environment condition. Therefore, the motor control of UAV has the characteristics of high reliability, high dynamic response, and high efficiency[2]. The control of the motor is essentially the control and adjustment of the speed of the motor. Motor control requires accurate rotor position and speed information, the traditional method of obtaining rotor information is to install mechanical sensors such as photoelectric encoders on the rotor shaft, prevalence of low reliability of this method, the restricted use conditions, anti-interference ability and high cost. Thus scholars study directed to a method of controlling the motor at a high reliability mechanical sensor cancellation condition, called the position sensorless control technology.

The specific method of position sensorless control technology is to extract the voltage and current signals in the motor windings, and extract the rotor speed and position information through appropriate methods, thereby replacing mechanical sensors. The most difficult point of this method lies in the estimation method of rotor speed and position. The existing position sensorless control methods are roughly divided into methods based on back-EMF observation and methods based on salient pole characteristic tracking [3-5].

When the motor is running in the low-speed area, the amplitude of the back EMF is too small to obtain accurate detection results. Therefore, methods based on salient pole characteristic tracking such as high-frequency signal injection are mainly used, and the estimated position and speed information of the motor rotor is obtained by using the salient pole effect of the motor rotor. For a surface permanent magnet synchronous motor, the inductance of the cross-axis and the direct-axis are equal, and there is no structural salient pole, therefore, it is necessary to construct a saturated salient pole when using this method. In addition, for some situations that do not require high accuracy, the I/F control method of speed-open-loop and current-closed-loop can be used to control the starting of the motor by controlling the amplitude of the stator winding current, so that the motor has a certain load torque matching ability and anti-load disturbance ability and does not depend on the motor parameters.

When the motor is running in the medium and high speed area, the commonly used position sensorless control methods include flux estimation method, sliding mode observer, model reference adaptive method, extended Kalman filter. The flux estimation method has a simple structure and fast dynamic response, but it relies on motor parameters. In practical applications, motor parameters are easily affected by factors such as environment,

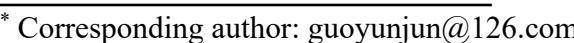


temperature, and speed. The sliding mode observer constructs a sliding mode variable structure system. Through the sliding mode control, the state quantity moves in a small amplitude on the sliding mode surface and finally reaches the equilibrium point, so as to realize the direct observation of the rotor speed and position information.

\section{The principle of I/F control}

Considering that the rotor motor does not need high observation accuracy when starting and running at low speed, a simple structure and easy-to-implement I/F control method can be adopted, which can start the rotor motor without detecting the initial position of the rotor [68]. During the operation of the motor, the $i_{d}^{*}=0$ control method is adopted, and the reference current $i_{d r e f}^{*}$ and $i_{\text {qref }}^{*}$ are both set to constants, and the frequency is a variable with a ramp change. If the ramp rate is $K_{\omega}$, and then

$$
\begin{aligned}
& \omega^{*}=\int K_{\omega} d t \\
& \theta^{*}=\int \omega^{*} d t
\end{aligned}
$$

The I/F control block diagram is shown in the figure. The reference position information obtained after two integrations is used for coordinate transformation. Therefore, different ramp rates can be selected according to the actual operating requirements of the rotor motor to start the motor in a suitable manner. Without the detection of the initial position of the rotor, the position angle $\theta^{*}$ determines a reference coordinate system $d^{*} q^{*}$, which is different from the actual coordinate system $d q$ by the angle $\theta_{L}$.During the starting process, $d^{*} q^{*}$ always lags behind $d q$, as shown in the figure.

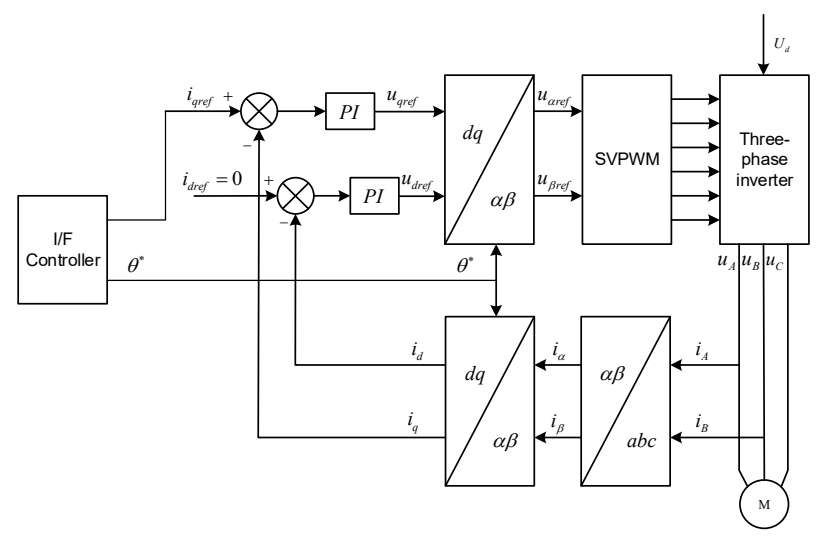

Fig. 1. I/F control principle block diagram

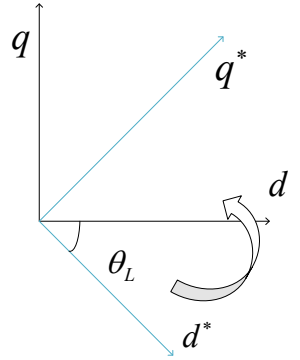

Fig. 2. Virtual coordinate system and actual coordinate system

The electromagnetic torque of PMSM can be expressed as follows:

$$
T_{e}=1.5 p \psi_{f} i_{q}^{*} \cos \theta_{L}
$$

The mechanical equation can be expressed as

$$
1.5 p \psi_{f} i_{q}^{*} \cos \theta_{L}-T_{L}=J \omega
$$

After the motor is started, the torque balance is reached at a certain moment, and the phase difference between the actual coordinate system and the virtual coordinate system is $\theta_{L}$, if the load torque is suddenly applied at this time, the motor torque $T_{e}$ is less than the load torque $T_{L}$. It can be seen from the mechanical equation that the rotor will decelerate to make the rotor angle closer to the given angle, and then the phase difference will decrease, the motor torque will increase, and finally a new torque balance will be achieved. In I/F control, in order to ensure the stable acceleration of the motor, the selection of the ramp rate is very important. If the rate of change is too small, the motor speed will rise slowly and fail to meet the requirements of fast response. If the rate of change is too large, the motor will lose step.

\section{Principle of sliding mode observer}

In the middle and high speed area of rotor motor operation, sliding mode observer is a highly robust control method. The principle of the sliding mode observer is based on the sliding mode variable structure control system. Determine an ideal sliding mode surface $s(x)=0$, select the appropriate switching function to make the moving point move in a small range on the predetermined sliding mode surface, and gradually reduce the moving range to reach the equilibrium point stably, so as to complete the tracking of the state of the system.

The system state quantity to be observed is the stator current. The back EMF quantity of the motor is extracted by function calculation, and the back EMF is processed through the phase-locked loop to obtain the estimated value of the rotor angle and position information.

The mathematical model of the permanent magnet synchronous motor in the $\alpha \beta$ coordinate system can be expressed as a form containing the back-EMF component[9],

$$
\left\{\begin{array}{l}
\frac{d i_{\alpha}}{d t}=-\frac{R}{L} i_{\alpha}+\frac{1}{L} u_{\alpha}-\frac{1}{L} e_{\alpha} \\
\frac{d i_{\beta}}{d t}=-\frac{R}{L} i_{\beta}+\frac{1}{L} u_{\beta}-\frac{1}{L} e_{\beta}
\end{array}\right.
$$


Where $e_{\alpha}, e_{\beta}$ is the back electromotive force component, $R$ is the stator resistance, and $\mathrm{L}$ is the stator inductance, then,

$$
\left\{\begin{array}{l}
e_{\alpha}=-k_{e} \omega \sin \theta \\
e_{\beta}=k_{e} \omega \cos \theta
\end{array}\right.
$$

Where $k_{e}$ is the back-EMF coefficient, $\omega$ is the rotor angular velocity, and $\theta$ is the rotor angle.

According to the above mathematical model, the following observer is constructed:

$$
\begin{aligned}
& \left\{\begin{array}{l}
\frac{d \hat{i}_{\alpha}}{d x}=-\frac{R}{L} \hat{i}_{\alpha}+\frac{1}{L}\left(u_{\alpha}-z_{\alpha}\right) \\
\frac{d \hat{i}_{\beta}}{d x}=-\frac{R}{L} \hat{i}_{\beta}+\frac{1}{L}\left(u_{\beta}-z_{\beta}\right)
\end{array}\right. \\
& \left\{\begin{array}{l}
z_{\alpha}=K_{1} \operatorname{sgn}\left(\hat{i}_{\alpha}-i_{\alpha}\right) \\
z_{\beta}=K_{1} \operatorname{sgn}\left(\hat{i}_{\beta}-i_{\beta}\right)
\end{array}\right.
\end{aligned}
$$

Among them, $K_{1}$ is the sliding mode switching gain, $z_{\alpha}, z_{\beta}$ is the current switching error signal, $\hat{i}_{\alpha}, \hat{i}_{\beta}$ is the observed value.

Subtract the motor model and the observer model to get the dynamic estimation deviation,

$$
\left\{\begin{array}{l}
\frac{d\left(\hat{i}_{\alpha}-i_{\alpha}\right)}{d x}=-\frac{R}{L}\left(\hat{i}_{\alpha}-i_{\alpha}\right)+\frac{1}{L}\left(e_{\alpha}-z_{\alpha}\right) \\
\frac{d\left(\hat{i}_{\beta}-i_{\beta}\right)}{d x}=-\frac{R}{L}\left(\hat{i}_{\beta}-i_{\beta}\right)+\frac{1}{L}\left(e_{\beta}-z_{\beta}\right)
\end{array}\right.
$$

Take the sliding surface ass $(i)=\left\{\begin{array}{l}\hat{i}_{\alpha}-i_{\alpha} \\ \hat{i}_{\beta}-i_{\beta}\end{array}\right.$, as long as the switching gain satisfies $K_{1}>\max \left(\left|e_{\alpha}\right|,\left|e_{\beta}\right|\right)$, there is $\mathrm{s}(i) \frac{\mathrm{ds}(i)}{\mathrm{dt}} \leq 0$, the observer can operate stably.

After a limited period of sliding mode movement, the sliding mode surface is $\mathrm{s}(i)=0$, then,

$$
\left\{\begin{array}{l}
e_{\alpha}=z_{\alpha}=K_{1} \operatorname{sgn}\left(\hat{i}_{\alpha}-i_{\alpha}\right) \\
e_{\beta}=z_{\beta}=K_{1} \operatorname{sgn}\left(\hat{i}_{\beta}-i_{\beta}\right)
\end{array}\right.
$$

It can be seen that the back-EMF is obtained by highfrequency switching of the current error, and the highfrequency switching is not continuous. The back-EMF obtained in this way has high-frequency interference signals, and a low-pass filter needs to be designed.

$$
\left\{\begin{array}{l}
\hat{e}_{\alpha}=\frac{\omega_{\text {cutoff }}}{s+\omega_{\text {cutoff }}} e_{\alpha} \\
\hat{e}_{\beta}=\frac{\omega_{\text {cutoff }}}{s+\omega_{\text {cutoff }}} e_{\beta}
\end{array}\right.
$$

Among them, $\omega_{\text {cutoff }}$ is the cutoff frequency of the filter.

The estimated position angle is as follows:

$$
\hat{\theta}_{1}=\tan ^{-1}\left(-\frac{\hat{e}_{\alpha}}{\hat{e}_{\beta}}\right)
$$

Since there is a phase delay of the low pass filter, and thus a counter electromotive force to estimate the rotor displacement there is a phase delay, so the increase in the estimated rotor position and a phase compensation,

$$
\hat{\theta}=\hat{\theta}_{1}+\Delta \theta
$$

Among, $\Delta \theta=\tan ^{-1}\left(\frac{\hat{\omega}}{\omega_{\text {cutoff }}}\right), \quad \hat{\omega}=\frac{\sqrt{\left(\hat{e}_{\alpha}\right)^{2}+\left(\hat{e}_{\beta}\right)^{2}}}{K_{e}}$.

The above analysis of the sliding mode observer is based on the ideal state, that is, once the moving point reaches the switching surface, it moves on the switching surface. In actual applications, after the moving point reaches the switching surface, it will continue to move through the switching surface for a period of time due to delays and other factors, and then return, and repeatedly traverse the switching surface in small amplitude, which is called system chattering. Chattering will affect the stability of the system, resulting in decreased accuracy or even instability. The principle of the sliding mode observer is based on the high-frequency switching of the switch, so the chattering cannot be completely eliminated, and it can only be weakened as much as possible by selecting an appropriate switching function. The literature [10-12]reduces chattering by choosing different switching functions instead of sign functions.

\section{Switching control algorithm}

The key technology to achieve permanent magnet synchronous motor control in accordance with the control method in the full speed range lies in the combination of low-speed and medium-high speed methods $[13,14]$. The appropriate switching algorithm is selected to achieve smooth switching between the two control methods. The determination of the upper and lower limits of the speed of the switching area depends on the reliable working area of the two control methods. The lower limit of the switching interval should be higher than the lowest speed for reliable operation of the sliding mode observer, and the upper limit should be higher than the highest speed for reliable operation of the $\mathrm{I} / \mathrm{F}$ control method. There are two main types of commonly used switching algorithms: hysteresis algorithm switching and weighted algorithm switching.

Weighting algorithm is a commonly used switching algorithm. The position and speed information of the rotor in the switching area are determined by the weighting of the estimated values of the two control methods. In theory, the smooth switching of the two control methods can be realized. The specific method is shown in the figure below, 


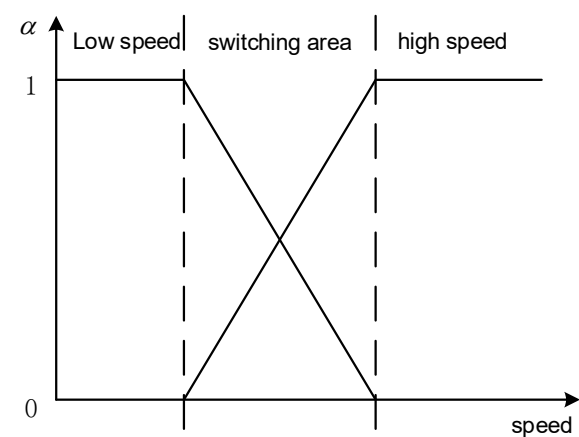

Fig. 3 Switching algorithm Schematic

The calculation formula for the position angle and $\hat{i}_{q}^{*}$ of the rotor in the switching area is as follows,

$$
\begin{aligned}
& \hat{\theta}_{r}=\alpha \hat{\theta}_{\text {before }}+(1-\alpha) \hat{\theta}_{\text {after }} \\
& \hat{i}_{q}^{*}=\alpha \hat{i}_{q_{-} \text {before }}^{*}+(1-\alpha) \hat{i}_{q_{-} \text {after }}^{*}
\end{aligned}
$$

The value of the weighting factor $\alpha$ is,

$$
\alpha=\left\{\begin{array}{l}
1, \hat{\omega}_{r} \leq \hat{\omega}_{\text {low }} \\
\frac{\hat{\omega}_{\text {high }}-\hat{\omega}_{r}}{\hat{\omega}_{\text {high }}-\hat{\omega}_{\text {low }}}, \hat{\omega}_{\text {low }}<\hat{\omega}_{r}<\hat{\omega}_{\text {high }} \\
0, \hat{\omega}_{r} \geq \hat{\omega}_{\text {high }}
\end{array}\right.
$$

\section{Simulation verification}

Table 1. The parameters of rotor motor

\begin{tabular}{ccc}
\hline \hline Parameter & Sign & Value \\
\hline Stator Resistance & $R_{s}$ & $0.008 \Omega$ \\
Pole pairs & $n_{p}$ & 5 \\
Stator Inductance & $L_{s}$ & $12 \mu \mathrm{H}$ \\
$\begin{array}{c}\text { Inertia } \\
\text { Permanent- } \\
\text { magnet flux }\end{array}$ & $J$ & $0.00347 \mathrm{~kg} \cdot \mathrm{m}^{2}$ \\
\hline \hline
\end{tabular}

To verify the performance of the composite control method of the rotor motor in the full speed range proposed in this paper, the SPMSM control system model was built and simulated. The specific motor parameters are shown in Table 1. The characteristic of the rotor motor is that the load torque changes with the speed of the motor. The fitting polynomial of the load torque changes with the motor is obtained by analyzing the data collected by the experiment. The total simulation time is $0.5 \mathrm{~s}$.

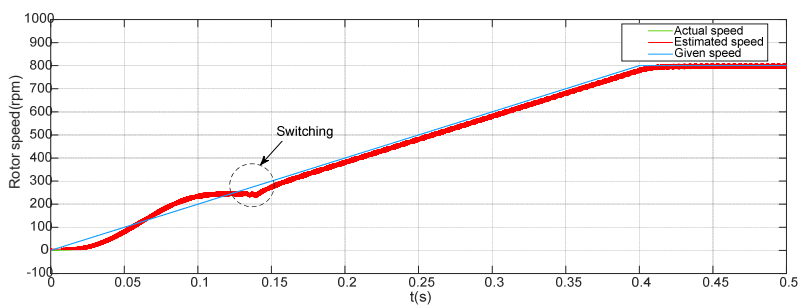

Fig. 4 Rotor speed estimation process

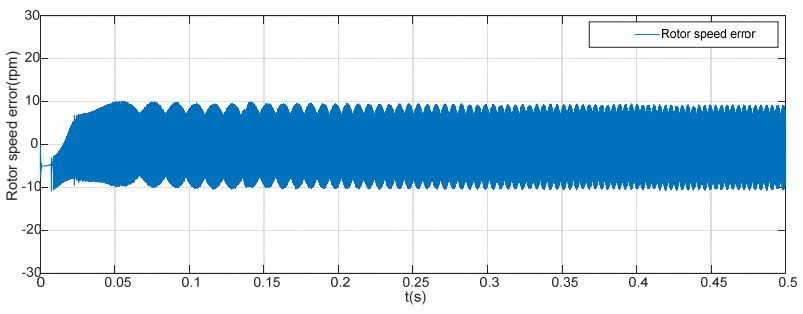

Fig. 5 Rotor speed estimation error

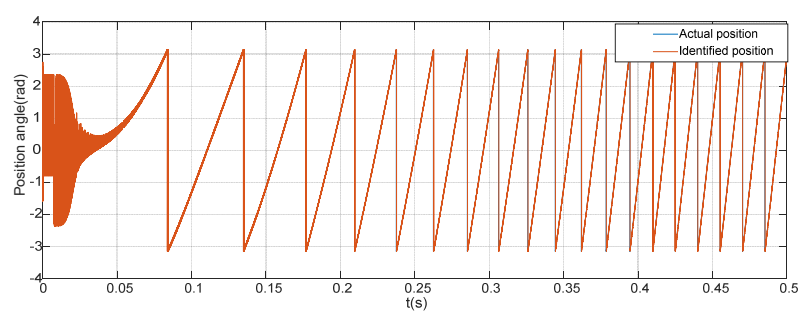

Fig. 6 Rotor position estimation process

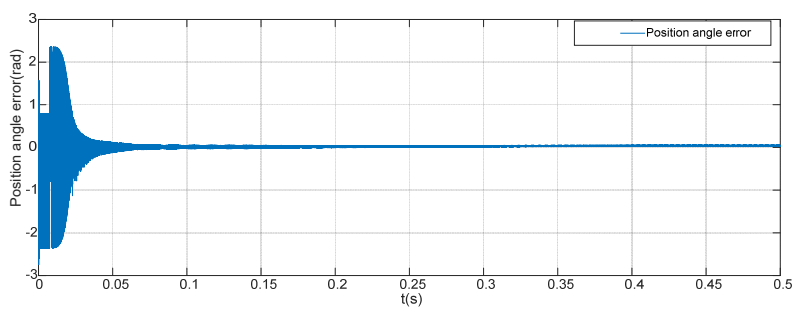

Fig. 7 Rotor position estimation error

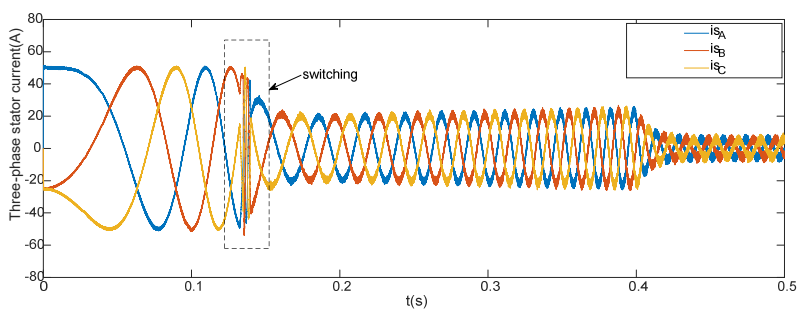

Fig. 8 Motor three-phase current waveform

The motor starts from zero speed through the $\mathrm{I} / \mathrm{F}$ control mode, it can be seen from Fig. 4 that there is a small range of fluctuations in the speed of the motor during the low-speed process. Since the drone has low requirements for position and speed accuracy in the start and low-speed areas, the fluctuations do not affect the actual operation of the motor. It can be seen from Fig. 6 and Fig. 7 that the sliding mode observer has a good tracking effect on the position of the motor rotor, and the chattering has a minimal effect on the stability of the system. The upper and lower limits of the rotation speed in the switching area are from 265 to $280 \mathrm{rpm}$. It can be seen from Fig. 4 and Fig. 6 that the weighted average algorithm realizes the gradual change of rotor speed and position angle, avoiding signal mutation and system imbalance.

Fig. 5 shows that the rotor speed estimation error does not exceed $\pm 10 \mathrm{rpm}$, and Fig. 7 shows that the rotor position estimation error is within $\pm 0.1 \mathrm{rad}$ when the speed is high enough. Both errors are within acceptable limits. The simulation results basically meet the needs of 
sensorless control within the full speed range of the rotor motor, and ensure smooth switching.

\section{Conclusion}

In this paper, a hybrid sensorless control method is proposed for the rotor motor of the UAV. It combines two control methods, namely, I/F control method and sliding mode observer, which are suitable for different speeds. An algorithm is designed to make sure the two control methods can be switched smoothly. Simulation results show that the hybrid control method has good control performance for the rotor motor of the UAV in the full speed range.

\section{References}

1. B. Fan, R. Zhang, Unmanned Aircraft System and Artificial Intelligence, Geomatics and Information Science of Wuhan University, 42 (2017) 1523-1529.

2. L. Zhao, K. Zhang, W. Wang, Position Control System Design for Muti-Rotor Type UAV, Computer Measurement\&Control, 24 (2016) 84-87.

3. J. Liu, F. Xiao, Y. Shen, Z. Mai, C. Li, PositionSensorless Control Technology of Permanent-Magnet Synchronous Motor-a Review, Transactions of China Electrotechnical Society, 32 (2017) 76-88.

4. Y. Liang, Y. Li, The State of art of Sensor $\neg$ less Vector Control of PMSM, Electric Drive, DOI (2003) 4-9.

5. G. Wang, M. Valla, J. Solsona, Position Sensorless Permanent Magnet Synchronous Machine Drives - A Review, IEEE Transactions on Industrial Electronics, 67 (2020) 5830-5842.

6. M. Wang, J. Yang, X. Zhang, C. Zhu, An I/f Control Method With Closed-loop Regulation of Current Vector for Surface Permanent Magnet Synchronous Motor Drives, Proceedings of the CSEE, 35 (2015) 2513-2521.

7. M. Wang, Y. Xu, J. Zou, H. Lan, An optimized IF startup method for BEMF-based sensorless control of SPMSM, 2017 IEEE Transportation Electrification Conference and Expo, Asia-Pacific (ITEC AsiaPacific), IEEE, 2017, pp. 1-6.

8. S.V. Nair, K. Hatua, N.D. Prasad, D.K. Reddy, A smooth and stable open-loop IF control for a surface mount PMSM drive by ensuring controlled starting torque, IECON 2018-44th Annual Conference of the IEEE Industrial Electronics Society, IEEE, 2018, pp. 355-360.

9. B. Wang, Y. Wang, Z. Wang, Direct torque control of permanent magnet synchronous motor drives using space vector modulation, Electric Machine and Control, 14 (2010) 45-50

10. Z. Hua, M. Dou, D. Zhao, L. Yan, J. Ji, J. Yang, Sensorless Control of Permanent Magnet Synchronous Motor Based on the Improved Sliding Mode Observer, Journal of Northwestern
Polytechnical University, 36 (2018) 754-760.

11. Y. Liao, J. Mo, L. Yan, Sensorless Control of Permanent Magnet Synchronus Motor Based on Improved Sliding Mode Observer, Small \& Special Electrical Machines, 48 (2020) 6-11.

12. Y. Zhang, D. Ding, X. Dong, Permanent Magnet Synchronous Motor Sensorless Control System Based on Sliding Mode Observer and Fuzzy PID, Electronic Measurement Technology, 41 (2018) 6-12.

13. J. Liu, F. Xiao Z. Mai, S. Gao, X. Yu, Hybrid PositionSensorless Control Scheme for PMSM Based on Combination of IF Control and Sliding Mode Observer, Transactions of China Electrotechnical Society, 33 (2018) 919-929.

14. W. Xu, The Realization of Permanent Magnet Synchronous Motor Vector Control, South China University of Technology, (2013). 